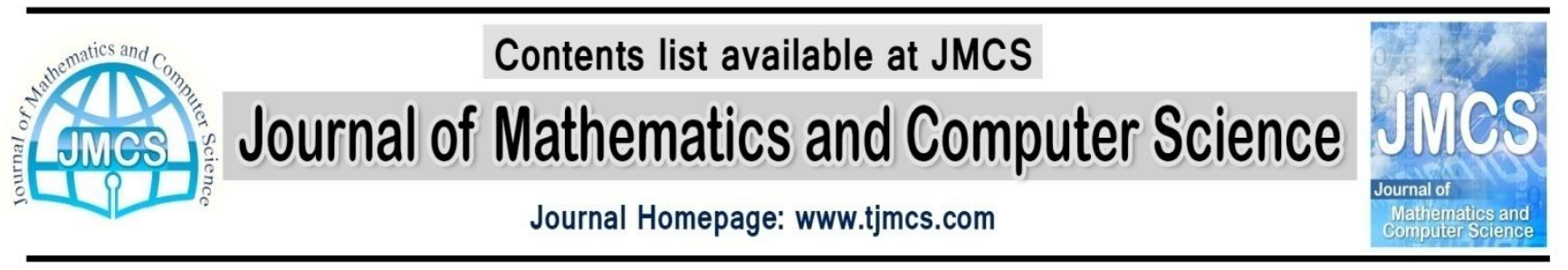

\title{
A Class of Multivalent Analytic Functions Defined by a New Linear Operator
}

\author{
Rahim Kargar ${ }^{1, *}$, Abdoljalil Bilavi ${ }^{2}$, Salahaddin Abdolahi ${ }^{1}$, Salah Maroufi ${ }^{1}$ \\ ${ }^{1}$ Department of Mathematics, Payame Noor University, I. R. of IRAN \\ ${ }^{2}$ Department of Mathematics, Shoush Branch, Islamic Azad University, Shoush, Iran \\ rkargar1983@gmail.com, jalil.math@yahoo.com, s.abdolahi2010@yahoo.com \\ maroufiheman@yahoo.com
}

\begin{abstract}
Article history:
Received May 2013

Accepted July 2013

Available online July 2013
\end{abstract}

\begin{abstract}
The main object of the present paper is to derive some results for multivalent analytic functions defined by a linear operator. Making use of a certain operator, which is defined here by means of Hadamard product, we introduce a subclasses $S_{A, B}^{p, \gamma}(\alpha, \lambda, \mu, v, a, c)$ of the class $A(p)$ of normalized $\mathrm{p}$-valent analytic functions on the open unit disk. Also we have extended some of the previous results and have given necessary and sufficient condition for this class.
\end{abstract}

Keywords: Analytic functions, Multivalent functions, Hadamard product, Subordination, Linear operators.

\section{Introduction}

Let $A(p)$ denote the class of functions $f$ of the form:

$$
f(z)=z^{p}+\sum_{k=1}^{\infty} a_{p+k} z^{p+k}
$$


$(p \in \mathbb{N}=\{1,2, \ldots\})$ which are analytic in the open unit disk $\Delta=\{z \in \mathbb{C}:|z|<1\}$. We write $A(1)=A$. If $f$ and $g$ are analytic in $\Delta$, we say that $f$ is subordinate to $g$ in $\Delta$, written $f \prec g$, if there exists Schwarz function $w$, analytic in $\Delta$ with $w(0)=0$ and $|w(z)|<1$ in $\Delta$ such that $f(z)=g(w(z)), z \in \Delta$. If $g$ is univalent and $g(0)=f(0)$, then $f(\Delta) \subset g(\Delta)$ it follows $f \prec g$. For two functions $f$ given by (1.1) and $g$ given by

$$
g(z)=\mathrm{z}^{\mathrm{p}}+\sum_{k=1}^{\infty} b_{p+k} z^{p+k}
$$

their Hadamard product (or convolution) is defined by

$$
(f * g)(z)=\mathrm{z}^{\mathrm{p}}+\sum_{k=1}^{\infty} a_{p+k} b_{p+k} z^{p+k} .
$$

For $a \in \mathbb{R}, c \in \mathbb{R} \backslash \mathbb{Z}_{0}^{-}$, where $\mathbb{Z}_{0}^{-}:=\{\ldots,-2,-1,0\}$, we introduce a linear operator

$$
\mathcal{J}_{\mu, \nu}^{\lambda, p}(a, c): A(p) \rightarrow A(p)
$$

defined by

$$
\mathcal{J}_{\mu, v}^{\lambda, p}(a, c) f(z)=\phi_{\mu, v}^{\lambda, p}(a, c ; z) * f(z), \quad(f \in A(p), z \in \Delta),
$$

where

$$
\phi_{\mu, v}^{\lambda, p}(a, c ; z)=z^{p}+\sum_{k=1}^{\infty} \frac{(a)_{k}(p+1)_{k}(p+1-\mu+v)_{k}}{(c)_{k}(p+1-\mu)_{k}(p+1-\lambda+v)_{k}} z^{p+k},
$$

and $(d)_{k}$ is the Pochhammer symbol defined by

$$
(d)_{k}= \begin{cases}1 & k=0 \\ d(d+1)(d+2) \ldots(d+k-1) & k \in \mathbb{N} .\end{cases}
$$

Also $0 \leq \lambda<1, \mu, v \in \mathbb{R}$ and $\mu-v-p<1$. We note that:

(i) If $\lambda=\mu=O$ in (1.2), then we have a linear operator was introduced by Saitoh [19].

(ii) If $a=c=1$ in (1.2), then $\phi_{\mu, v}^{\lambda, p}(a, c ; z) * f(z) \equiv \Delta_{z, p}^{\lambda, \mu, v} f(z)$, where $\Delta_{z, p}^{\lambda, \mu, v} f(z)$ is the fractional operator introduced by Choi[6]. 
We now introduce the following family of linear operators $\mathcal{L}_{\mu, v}^{\lambda, p, \alpha} f(z)$ analogous to $\mathcal{J}_{\mu, \nu}^{\lambda, p}(a, c)$ :

$$
\mathcal{L}_{\mu, v}^{\lambda, p, \alpha}(a, c): A(p) \rightarrow A(p)
$$

which defined as

$$
\mathcal{L}_{\mu, \nu}^{\lambda, p, \alpha}(a, c) f(z):=\psi_{\mu, \nu}^{\lambda, p, \alpha}(a, c ; z) * f(z),
$$

where $\psi_{\mu, v}^{\lambda, p, \alpha}(a, c ; z)$ is the function defined in terms of the Hadamard product by the following condition:

$$
\phi_{\mu, \nu}^{\lambda, p}(a, c ; z) * \psi_{\mu, v}^{\lambda, p, \alpha}(a, c ; z)=\frac{z^{p}}{(1-z)^{\alpha+p}}, \quad(\alpha>-p) .
$$

We can easily find from (1.3)-(1.5) that

$$
\mathcal{L}_{\mu, v}^{\lambda, p, \alpha}(a, c) f(z)=z^{p}+\sum_{k=1}^{\infty} \frac{(c)_{k}(p+1-\mu)_{k}(p+1-\lambda+v)_{k}(\alpha+p)_{k}}{(a)_{k}(p+l)_{k}(p+1-\mu+v)_{k} k !} a_{p+k} z^{p+k} .
$$

By definition and specializing the parameters $\lambda, \mu, p, a, c$ and $\alpha$ we obtain: $\mathcal{L}_{0, v}^{0, p, 1}(p+1,1) f(z)=f(z) \quad$ and $\quad \mathcal{L}_{0, v}^{0, p, 1}(p, 1) f(z)=z f^{\prime}(z) / p$. It should be remarked that the linear operator $\mathcal{L}_{\mu, v}^{\lambda, p, \alpha}(a, c) f(z)$ is a generalization of many other linear operators considered earlier. In particular, for $f \in A(p)$, we have the following observations:

- $\mathcal{L}_{0, v}^{0, p, \alpha}(a, c) f(z) \equiv \mathcal{J}_{p}^{\alpha}(a, c) f(z)$, the Cho-Kwon-Srivastava operator [5].

- $\mathcal{L}_{0, v}^{0, p, \alpha}(a, a) f(z) \equiv \mathcal{D}^{\alpha+p-1} f(z)$, where $\mathcal{D}^{\alpha+p-1}$ is the Well-known Ruscheweyh derivative of $(\alpha+p-1)$-th order was studied by Goel and Sohi [9].

- $\mathcal{L}_{0, v}^{0, p, 1}(p+1-\lambda, 1) f(z) \equiv \Omega_{z}^{(\lambda, p)}=\frac{\Gamma(p+1-\lambda)}{\Gamma(p+1)} z^{\lambda} \mathcal{D}_{z}^{\lambda} f(z)$, where $\Omega_{z}^{(\lambda, p)}$ is the fractional derivative operator defined by Srivastava and Aouf [20] and $\mathcal{D}_{z}^{\lambda} f(z)$ is the fractional derivative of $f(z)$ of order $\lambda[12,15,17]$.

- $\mathcal{L}_{0, v}^{0,1, \alpha-1}(a, c) f(z) \equiv \mathcal{J}_{c}^{a, \alpha} f(z)$, the linear operator investigated by Hohlov [11]. 
- $\quad \mathcal{L}_{0, v}^{0,1-\alpha, \alpha}(a, c) f(z) \equiv \mathcal{L}_{p}(a, c) f(z)$, the linear operator studied by Saitoh [19] which yields the operator $\mathcal{L}(a, c) f(z)$ introduced by Carleson and Shaffer for $p=1$ [3].

- $\mathcal{L}_{0, v}^{0, p, \alpha}(\alpha+p+1,1) f(z) \equiv \mathcal{F}_{\alpha, p}(f)(z)=\frac{\alpha+p}{z^{\alpha}} \int_{0}^{z} t^{\alpha-1} f(t) d t,(\alpha>-p)$, the generalized Bernardi-Libera-Livingston integral operator [7].

- $\mathcal{L}_{0, v}^{0,1-\alpha, \alpha}(\lambda+1, \mu) f(z) \equiv \mathcal{J}_{\lambda, \mu} f(z),(\lambda>-1, \mu>0)$, the Choi-SaigoSrivastava operator which is closely related to the Carleson-Shaffer operator $\mathcal{L}(\mu, \lambda+1) f(z)[3]$.

- $\mathcal{L}_{0, v}^{0, p, 1}(p+\alpha, 1) f(z) \equiv \mathcal{J}_{\alpha, p} f(z),(\alpha \in \mathbb{Z}, \alpha>-p)$, the operator considered by Liu and Noor [13].

Now by using the linear operator $\mathcal{L}_{\mu, v}^{\lambda, p, \alpha}(a, c) f(z)$, defined by (1.6), we introduce the new class $S_{A, B}^{p, \gamma}(\alpha, \lambda, \mu, v, a, c)$ as follows:

Definition 1.1. We say that a function $f \in A(p)$ is in the class $S_{A, B}^{p, \gamma}(\alpha, \lambda, \mu, v, a, c)$, if it satisfies the following condition:

$$
\frac{1}{p-\gamma}\left[\frac{\left(\mathcal{L}_{\mu, \nu}^{\lambda, p, \alpha}(a, c) f(z)\right)^{\prime}}{z^{p-l}}-\gamma\right] \prec \frac{1+A z}{1+B z}, \quad z \in \Delta,
$$

where $-1 \leq B<A \leq 1$ and $0 \leq \gamma<p$.

By specializing the parameters $A, B, \alpha, \lambda, \mu, \gamma, a, c$ and $p$, we obtain many classes which were studied by authors earlier. For more details see $[1,2,4,10,12,14,15]$. The aim of this paper, is to give more results the above class of multivalent functions. Also we continue and extended some of the previous results and have given other properties of this class.

\section{Main Results}

Theorem 2.1. A function $f \in A(p)$ belongs to the class $S_{A, B}^{p, \gamma}(\alpha, \lambda, \mu, v, a, c)$ if and only if

$$
\frac{(f * \mathcal{X})(z)}{z^{p}}+\frac{z}{p}\left[\frac{(f * \mathcal{X})(z)}{z^{p}}\right]^{\prime} \prec \frac{1+A z}{1+B z}, \quad z \in \Delta,
$$

where 
$\mathcal{X}:=z^{p}+\frac{p}{p-\gamma} \sum_{k=1}^{\infty} \frac{(c)_{k}(p+1-\mu)_{k}(p+1-\lambda+v)_{k}(\alpha+p)_{k}}{(a)_{k}(p+1)_{k}(p+1-\mu+v)_{k} k !} z^{p+k}$.

Proof. Let $f \in A(p)$. From (1.7) we have

$$
\begin{aligned}
& \frac{1}{p-\gamma}\left[\frac{\left(\mathcal{L}_{\mu, \nu}^{\lambda, p, \alpha}(a, c) f(z)\right)^{\prime}}{z^{p-1}}-\gamma\right] \\
& =\frac{1}{p-\gamma}\left[p z^{p-1}+\sum_{k=1}^{\infty} \frac{(c)_{k}(p+1-\mu)_{k}(p+1-\lambda+v)_{k}(\alpha+p)_{k}}{(a)_{k}(p+1)_{k}(p+1-\mu+v)_{k} k !}(p+k) a_{p+k} z^{p+k-1} / z^{p-1}-\gamma\right] \\
& =1+\sum_{k=1}^{\infty} \frac{(c)_{k}(p+1-\mu)_{k}(p+1-\lambda+v)_{k}(\alpha+p)_{k}(p+k)}{(p-\gamma)(a)_{k}(p+l)_{k}(p+1-\mu+v)_{k} k !} a_{p+k} z^{k} \\
& =\left[1+\sum_{k=1}^{\infty} a_{p+k} z^{k}\right] *\left[1+\sum_{k=1}^{\infty} \frac{(c)_{k}(p+1-\mu)_{k}(p+1-\lambda+v)_{k}(\alpha+p)_{k}(p+k)}{(p-\gamma)(a)_{k}(p+1)_{k}(p+1-\mu+v)_{k} k !} z^{k}\right] \\
& =\frac{f(z)}{z^{p}} *\left[\frac{x}{z^{p}}+z p\left(\frac{x}{z^{p}}\right)^{\prime}\right] \\
& =\frac{(f * X)(z)}{z^{p}}+\frac{z}{p}\left[\frac{(f * X)(z)}{z^{p}}\right]^{\prime} \prec \frac{1+A z}{1+B z}, \quad z \in \Delta,
\end{aligned}
$$

and therefore the left-hand side of (1.7) and of (2.1) are the same.

If we put $\alpha=c=1, \lambda=\mu=0$ and $a=p+1$ in theorem 2.1, we have:

Corollary 2.1. A function $f \in A(p)$ belong to $S_{p}(A, B, \gamma)$ (see [2]) if and only if

$$
1+\frac{1}{p-\gamma} \sum_{k=1}^{\infty}(p+k) a_{p+k} z^{k} \prec \frac{1+A z}{1+B z} .
$$

If we put $\alpha=A=c=1, \lambda=\mu=0, B=-1$ and $a=p+1$ in theorem 2.1, we have:

Corollary 2.2. A function $f \in A(p)$ belong to $S_{p}(\gamma)$ (see [15]) if and only if

$$
1+\frac{1}{p-\gamma} \sum_{k=1}^{\infty}(p+k) a_{p+k} z^{k} \prec \frac{1+z}{1+z} \text {. }
$$

If we put $\alpha=c=1, \lambda=\mu=\gamma=0$ and $a=p+1$ in theorem 2.1, we have: 
Corollary 2.3. A function $f \in A(p)$ belong to $S_{p}(A, B)$ (see [4]) if and only if

$$
1+\frac{1}{p} \sum_{k=1}^{\infty}(p+k) a_{p+k} z^{k} \prec \frac{1+A z}{1+B z}
$$

If we put $A=a=c=1, \lambda=\mu=0, B=-1$ and $\alpha=n$ in theorem 2.1, we have:

Corollary 2.4. A function $f \in A(p)$ belong to $\mathcal{T}_{n+p-1}(\gamma)$ (see [10]) if and only if

$$
1+\frac{1}{p-\gamma} \sum_{k=1}^{\infty} \frac{(p+k)(n+p)_{k}}{k !} a_{p+k} z^{k} \prec \frac{1+z}{1+z} \text {. }
$$

If we put $a=c=1, \lambda=\mu=\gamma=0, A \rightarrow-A, B \rightarrow-B$ and $\alpha=n$ in theorem 2.1, we have:

Corollary 2.5. A function $f \in A(p)$ belong to $\mathcal{V}_{n, p}(A, B)$ (see [12]) if and only if

$$
1+\frac{1}{p} \sum_{k=1}^{\infty} \frac{(p+k)(n+p)_{k}}{k !} a_{p+k} z^{k} \prec \frac{1-A z}{1-B z} \text {. }
$$

If we put $a=c=1, \lambda=\mu=0, A \rightarrow-A, B \rightarrow-B$ and $\alpha=n$ in theorem 2.1, we have:

Corollary 2.6. A function $f \in A(p)$ belong to $\mathcal{V}_{n, p}(A, B, \gamma)$ (see [1]) if and only if

$$
1+\frac{1}{p-\gamma} \sum_{k=1}^{\infty} \frac{(p+k)(n+p)_{k}}{k !} a_{p+k} z^{k} \prec \frac{1-A z}{1-B z} \text {. }
$$

If we put $\alpha=c=p=1, \lambda=\mu=\gamma=0, A \rightarrow-A, B \rightarrow-B$ and $\alpha=p+1$ in theorem 2.1, we have:

Corollary 2.7. A function $f \in A(p)$ belong to $\mathcal{K}(A, B)$ (see [14]) if and only if

$$
1+\sum_{k=1}^{\infty}(1+k) a_{1+k} z^{k} \prec \frac{1-A z}{1-B z} .
$$

Theorem 2.2. A function $f$ belongs to the class $S_{A, B}^{p, \gamma}(\alpha, \lambda, \mu, \nu, a, c)$ if and only if there exists a Schwarz function $w(z)$ such that

$$
f(z)=\left[\sum_{k=1}^{\infty} \frac{1}{\mathcal{Q}(k)}\left(\frac{k}{p+k}\right) z^{p+k}\right] * z^{p}\left[\int_{z}\left(\frac{\gamma}{t}+\frac{p-\gamma}{t} \frac{1+A w(t)}{1+B w(t)}\right) d t-\operatorname{Ln} z^{p}\right]
$$

where 


$$
\mathcal{Q}(k):=\frac{(c)_{k}(p+1-\mu)_{k}(p+1-\lambda+v)_{k}(\alpha+p)_{k}}{(a)_{k}(p+1)_{k}(p+1-\mu+v)_{k} k !}
$$

Proof. Let $f \in S_{A, B}^{p, \gamma}(\alpha, \lambda, \mu, v, a, c)$. Then from the definition 1.1, we have

$$
\frac{1}{p-\gamma}\left[\frac{\left(\mathcal{L}_{\mu, \nu}^{\lambda, p, \alpha}(a, c) f(z)\right)^{\prime}}{z^{p-1}}-\gamma\right]=\frac{1+A w(z)}{1+B w(z)}, \quad z \in \Delta,
$$

where $|w(z)|<1$ in $\Delta$ with $w(0)=0$. Therefore

$$
\begin{aligned}
& \frac{\left(\mathcal{L}_{\mu, v}^{\lambda, p, \alpha}(a, c) f(z)\right)^{\prime}}{Z^{p}}=\frac{\gamma}{Z}+\frac{(p-\gamma)}{Z} \frac{1+A w(z)}{1+B w(z)} \\
& \Rightarrow \int_{z} \frac{\left(\mathcal{L}_{\mu, v}^{\lambda, p, \alpha}(a, c) f(t)\right)^{\prime}}{t^{p}} d t=\int_{z}\left[\frac{\gamma}{t}+\frac{(p-\gamma)}{t} \frac{1+A w(t)}{1+B w(t)}\right] d t \\
& \Rightarrow \frac{\mathcal{L}_{\mu, \nu}^{\lambda, p, \alpha}(a, c) f(z)}{z^{p}}+\operatorname{Ln} z^{p}+\sum_{k=1}^{\infty} \mathcal{Q}(k) \frac{p}{k} a_{p+k} z^{k} \\
& =\int_{z}\left[\frac{\gamma}{t}+\frac{(p-\gamma)}{t} \frac{1+A w(t)}{1+B w(t)}\right] d t .
\end{aligned}
$$

Thus from (1.4) and (1.6) we obtain

$$
f(z) * \sum_{k=1}^{\infty} \mathcal{Q}(k)\left[\frac{p+k}{k}\right] z^{p+k}=z^{p}\left[\int_{z}\left[\frac{\gamma}{t}+\frac{(p-\gamma)}{t} \frac{1+A w(t)}{1+B w(t)}\right] d t-\operatorname{Ln} z^{p}\right],
$$

and our assertion follows immediately.

Remark 2.1. By specializing the parameters $A, B, \alpha, \lambda, \mu, \gamma, a, c, p$ and by using Theorem 2.2 and Corollaries 2.1-2.7, we get necessary and sufficient condition for functions belongs to classes were studied in $[1,2,4,10,12,14,15]$.

\section{References}

[1] M.K. Aouf, A generalization of multivalent functions defined by Ruscheweyh derivatives, Soochow J. Math. 17(1991)83-97.

[2] M.K. Aouf, On certain subclass of analytic $p$-valent function. II, Math. Japan. 34(1989)683-691. 
[3] B.C. Carlson and D. B. Shaffer, Starlike and prstarlike hypergeometric functions, SIAM J. Math. Anal. 15(1984)737-745.

[4] M.P. Chen, A class of $p$-valent functions, Soochow J. Math. 8(1982)15-26.

[5] N.E. Cho, O.H. Kwon and H. M. Srivastava, Inclusion and argument properties for certain subclasses of multivalent functions associated with a family of linear operators, J. Math. Anal. Appl. 292(2004)470-483.

[6] Jae Ho Choi, On differential subordinations of multivalent functions involving a certain fractional derivative operator, International Journal of Mathematics and Mathematical Sciences, (2010)1-10.

[7] J.H. Choi, M. Saigo and H. M. Srivastava, Some inclusion properties of a certain family of integral opertaors, J. Math. Anal. Appl. 276 (2002)432-445.

[8] J. Dziok, Classes of analytic funcions involving some integral operator, FoliaSci. Univ. Tech. Resoviensis 20(1995)21-39.

[9] R.M. Goel and N.S. Sohi, A new criterion for $p$-valent functions, Proc. Amer. Math. Soc. 78(1980) 353-357.

[10] R.M. Goel and N.S. Sohi, New criteria for $p$-valence, Indian J. Pure Appl. Math. 11(1980)1356-1360.

[11] Yu. E. Hohlov, Operators and operations in the class of univalent functions, Izv, Vvssh. Ucebn. Zaved. Math. 10(1987) 83-89(in Russian)

[12] V. Kumar and S.L. Shukla, Multivalent function defined by Ruscheweyh derivatives, II, Indian J. Pure Appl. Math. 15(1984)1228-1238.

[13] J.L. Liu and K.I. Noor, Some properties of Noor integral operator, J. Natur. Geom. 21(2002)81-90.

[14] B.S. Mehrok, A class of univalent functions, Tamkang J. Math 13(1982)141155 .

[15] S. Owa, On certain subclass of analytic $p$-valent function. Math. Japon. 29(1984)191-198. 
[16] S. Owa, On the distortion theorems, I. Kyungpook Math. J. 18(1978)53-59.

[17] S. Owa, H. M. Srivastava, Univalent starlike generalized hypergeometric functions, Canad. J. Math. 39(1987)1057-1077.

[18] S. Rusheweyh, New criteria for univalent functions, Proc. Amer. Math. Soc. 49(1975) 109-115.

[19] H. Saitoh, A liner operator and its applications of first order deffrential subodinations, Math. Japon. 44(1996)31-38.

[20] H.M. Srivastava and M.K. Aouf, A certain fractional derivative operator and its applications to a new class of analytic and multivalent functions with negative coefficients. I. J. of Mathematical Analysis and Applications, 171(1992) 1-13. 